\title{
РИСК ОСТЕОПОРОТИЧЕСКИХ ПЕРЕЛОМОВ У ПАЦИЕНТОВ, ПРОХОДЯЩИХ МЕДИЦИНСКУЮ РЕАБИЛИТАЦИЮ
}

\author{
Марченкова Л.А., Макарова Е.В.
}

ФГБУ «Национальный медицинский исследовательский центр реабилитации и курортологии» Министерства здравоохранения Российской Федерации

ЦЕЛЬ: изучить распространенность остеопороза (ОП) и низкотравматических переломов, а также оценить абсолютный риск переломов среди пациентов старше 50 лет, проходящих медицинскую реабилитацию.

МАТЕРИАЛЫ И МЕТОДЫ: Проведено поперечное исследование путем анкетного опроса мужчин и женщин в возрасте 50 лет и старше, госпитализированных в ФГБУ «НМИЦ РК» Минздрава России для прохождения курса медицинской реабилитации.

У всех пациентов, включенных в исследование, был собран анамнез жизни и заболевания. Абсолютная 10-летняя вероятность основных остеопорозных переломов рассчитывалась по российской модели калькулятора FRAX.

PЕзУЛЬтАТЫ: с учетом качества заполненных данных, критериев включения и не включения в исследование, в статистический анализ были включены анкеты 600 пациентов (174 мужчин и 426 женщин).

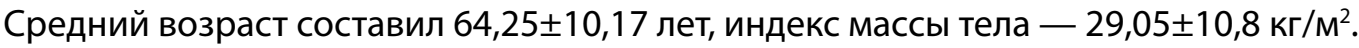

47,5\% (n=285) участников проходили медицинскую реабилитацию по поводу заболеваний опорно-двигательного аппарата, 233 пациента (38,8\%) по поводу соматической патологии и 13,4\% (n=81) по профилю «нейрореабилитация».

Треть опрошенных пациентов имела высокий риск переломов: 224 человека (38\% всех опрошенных, у 45,7\% женщин n=195 и 16,6\% мужчин n=29). Средний 10-ти летний риск для основных остеопорозных переломов составил 13,7\% [1,6;48]. У 8,6\% пациентов $(\mathrm{n}=52)$ значение абсолютного риска переломов по модели FRAX было выше 30\%.

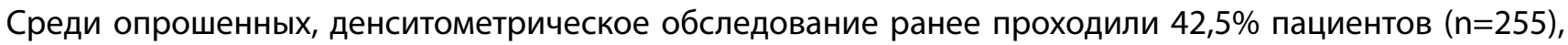
однако менее половины (18,16\% общего числа опрошенных, $\mathrm{n}=109)$ - костную денситометрию диагностически значимых отделов осевого скелета. Ранее слышали от врачей, что они имеют высокий риск пе-

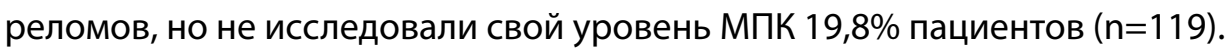

У $34,1 \%(n=205)$ пациентов в исследуемой группе ранее уже был установлен диагноз системного ОП. Длительность с момента верификации заболевания у этих больных составила от 6 месяцев до 20 лет,

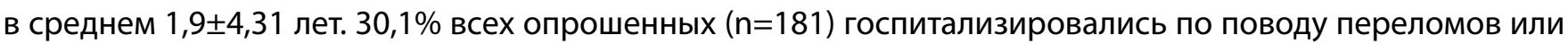
ОП как минимум один раз за последние пять лет. 45,8\% респондентов $(\mathrm{n}=275)$ перенесли когда-либо низкотравматические переломы, причем по данным анамнеза, у 13 из них (4,6\%) патологический перелом возник на фоне проводимых ранее процедур массажа, мануальной терапии, лечебной физкультуры или механотерапии.

120 пациентов, что составило 20\% от всех опрошенных и 58,5\% от пациентов с установленным ранее диагнозом ОП, на момент госпитализации в реабилитационный стационар получали патогенетическую терапию. Однако в 69,0\% случаев терапия осуществлялась только препаратами кальция и/или витамина D, и лишь 31,0\% пациентов с ОП принимали антирезорбтивный или костноанаболический препарат. На момент опроса продолжало какую-либо терапию ОП 74 человека (12,3\% всех испытуемых).

ВЫВОДЫ: результаты исследования продемонстрировали высокую распространенность ОП и низкоэнергетических переломов среди пациентов старше 50 лет, проходящих медицинскую реабилитацию. 38\% таких пациентов имеют высокий 10-ти летний риск остеопорозных переломов составил, из которых у 8,6\% этот риск был выше 30\%. Выявлена недостаточная частота назначения эффективной патогенетической терапии остеопороза - только у 58,5\% от пациентов с установленным ранее диагнозом ОП. 